\title{
Parametric signal fitting of highly asymmetric voltammograms by using the exponentially modified gaussian (EMG) function
}

Santiago Cavanillas, Núria Serrano, José Manuel Díaz-Cruz*, Cristina Ariño, Miquel Esteban.

Departament de Química Analítica. Facultat de Química. Universitat de Barcelona. Martí i Franquès 1-11, E-08028-Barcelona (Spain).

*: Corresponding author

Tel.: 34 934039116, Fax: 34 934021233, e-mail: josemanuel.diaz@ub.edu

\begin{abstract}
A new multivariate curve resolution method is proposed according to the parametric signal fitting (PSF) strategy for the analysis of overlapping and highly asymmetric voltammetric signals. The method is based on the fitting of the well-known exponentially modified Gaussian (EMG) function, which had been previously used for the resolution of coeluted chromatographic peaks. The main advantage of this function is that one of the adjustable parameters is just the area under the peak, which is a magnitude more reliable that the peak height in the case of electrochemical systems providing highly asymmetric voltammograms. After a preliminary test with simulated data, the proposed method has been successfully applied to the experimental system Cd(II)-Cysteine in both mercury and carbon screen-printed electrodes.
\end{abstract}

Keywords: Voltammetry; Multivariate curve resolution (MCR); Non-linearity; exponentially modified gaussian (EMG); Asymmetric peak; 


\section{Introduction}

Despite the intricate character of their signals, voltammetric techniques are useful in many situations due to their low cost, experimental simplicity, versatility and high sensitivity [1]. In complicated systems yielding strongly overlapping peaks, chemometric methods may be helpful to resolve the different contributions of the electrochemical processes to the measured voltammograms [2-3]. If such contributions are linear, techniques like partial least squares (PLS) or multivariate curve resolution by alternating least squares (MCR-ALS) provide good results [3]. However, most electrochemical processes are far from linearity and usually produce datasets with signals changing its shape and/or moving along the potential axis as the experiment takes place. Then, the application of linear chemometric methods like PLS or MCRALS yield unrealistic results affected by a large error.

In the study of non-linear systems, artificial neural networks (ANN) are very effective, but they require extensive calibration datasets [4]. An alternative approach is parametric signal fitting (PSF), which uses functions with adjustable parameters to reproduce the shape and position of voltammetric signals along the experiments [5-8]. The values of such parameters can be further related to some properties of the electroactive species like the concentration, the peak potential or the half-peak width. The first function to be used according to PSF strategy was a set of two gaussian peaks sharing the same position and height but with different widths at both sides of the maximum. This simple approach, called gaussian peak adjustment (GPA), was successfully applied to relatively symmetric voltammograms [5] and was improved with the implementation of transversal constraints [7]. The main problem of the double gaussian function of GPA is that the fitting in the vicinity of the maximum is not good for strongly asymmetric signals like these obtained in linear sweep (LSV) or cyclic (CV) voltammetries. 
Voltammograms measured for irreversible systems presented similar fitting drawbacks. This is why a more complex function like asymmetric logistic peak was tried in the ALPA method [8]. Although in many cases the fitting was very good, the complexity of the function required a high accuracy in the selection of initial estimations for the adjustable parameters, resulting in a poor robustness when highly overlapping or noisy data had to be analysed.

In the present work, a new method using an asymmetric function, simpler than ALPA but still accurate around the maximum, is developed and tested in different voltammetric data. It is called parametric signal fitting by exponentially modified gaussian (PSF-EMG). The EMG function has been frequently used to deconvolute peaks in liquid chromatography [9-12], but, as far as we know, not yet in the analysis of voltammetric data. A remarkable characteristic of this function is that one of the adjustable parameters is just the area under the peak, which can be very useful in the treatment of irreversible voltammetric signals, where the area is more representative of the concentration than the signal height. Besides, that this algorithm permits the adjustment of strongly asymmetric peaks. Firstly, EMG method will be applied to a simulated set of voltammetric data, and secondly, it will be validated with experimental voltammetric data obtained from the well-known $\mathrm{Cd}(\mathrm{II})$-cysteine system using both a mercury electrode (SMDE) and a screen printed carbon electrode (SPCE). The results obtained by applying the EMG method will be compared with those computed by using the previous GPA and ALPA approaches.

\section{Theory}

The exponentially modified gaussian (EMG) function can be written in the form:

$$
I=\frac{a}{2 d} \exp \left(\frac{c^{2}}{2 d^{2}}-\frac{E-b}{d}\right) \operatorname{erfc}\left(\frac{1}{\sqrt{2}}\left(\frac{c}{d}-\frac{E-b}{c}\right)\right)
$$


where ' $I$ ' is the current, ' $E$ ' is the potential, ' $a$ ' is the area under the peak, ' $b$ ' is a potential value close (but not equal) to that of the maximum, ' $c$ ' is related to the peak width and ' $d$ ' is related to the peak symmetry. The term 'erfc' denotes the complementary error function. This is the usual form of the EMG equation to be used in the fitting of chromatography peaks, with the queue at the right side of the maximum. However, in voltammetry the queue can be also at the left side. Then, a modified form of Eqn.(1) can be applied:

$$
I=\frac{a}{2 d} \exp \left(\frac{c^{2}}{2 d^{2}}+\frac{E-b}{d}\right) \operatorname{erfc}\left(\frac{1}{\sqrt{2}}\left(\frac{c}{d}+\frac{E-b}{c}\right)\right)
$$

The functions described by Eqns. (1) and (2) are symmetric with respect to the line where $E=b$ and where both expressions reach the same $I$ value.

For quite symmetric peaks, the fitting of the previous EMG functions is slow and not very accurate, so that the GPA function can be a better option:

$$
\begin{array}{ll}
I=a \exp \left(-c(E-b)^{2}\right) & \text { if } E<b \\
I=a \exp \left(-d(E-b)^{2}\right) & \text { if } E \geq b
\end{array}
$$

where ' $a$ ' is now the peak height, ' $b$ ' is the peak potential and ' $c$ ' and ' $d$ ' are related to the peak width at the left side and the right side of the maximum, respectively. Figure 1 compares the shapes of the functions described by Eqns. (1)-(3), that in the following we will call EMG(-), EMG(+) and GPA, respectively.

Figure 2 summarises the structure of the method PSF-EMG which adjusts by least squares the experimental voltammograms to the previous equations by means of several home-made functions implemented in Matlab [13] and freely available at the website http://www.ub.edu/dqaelc/programes_eng.html. It must be remarked the initial estimate of the half-peak widths at both sides of every maximum, which allows the program to select the most appropriate equation depending on the ratio between such widths. As a result of the application of the method, concentration profiles can be obtained that can 
be used to determine the stoichiometry of the formed complexes and, in the most favourable situations, could be fitted to complexation models to estimate the corresponding stability constants, as it is made in reference [14]. The accuracy of the fitting is analysed in terms of the lack of fit (lof), defined as:

$$
\operatorname{lof}(\%)=\sqrt{\frac{\sum_{i j}\left(x_{i j}-\hat{x}_{i j}\right)^{2}}{\sum_{i j}\left(x_{i j}\right)^{2}}} \times 100
$$

where $x_{i j}$ are the elements of the experimental data matrix and $\hat{x}_{i j}$ are the elements of the reproduced data matrix with the optimised EMG parameters.

\section{Material and Methods}

\subsection{Chemicals and instrumentation}

$\mathrm{Cd}(\mathrm{II}) \quad 0.01 \mathrm{~mol} \mathrm{\textrm {L } ^ { - 1 }}$ stock solutions were prepared from $\mathrm{Cd}\left(\mathrm{NO}_{3}\right)_{2} \cdot 4 \mathrm{H}_{2} \mathrm{O}$ (Merck, analytical grade) and standardized by complexometric titration with EDTA at $\mathrm{pH} 5.5$ using hexamine and xylenol orange as indicator. A stock solution of $10^{-3} \mathrm{~mol} \mathrm{~L}^{-1}$ of Lcysteine (Cys) (Aldrich, >99.0\%) was prepared by dissolving the reagents in water. 0.02 mol L ${ }^{-1}$ Tris(hydroxymethyl)aminomethane (TRIS) buffer was prepared at $\mathrm{pH} 7.5$ and $0.05 \mathrm{~mol} \mathrm{~L}^{-1} \mathrm{KNO}_{3}$ ionic strength. $0.1 \mathrm{~mol} \mathrm{~L}^{-1} \mathrm{HNO}_{3}$ and $0.1 \mathrm{~mol} \mathrm{~L}^{-1} \mathrm{KOH}$ solutions were prepared from Titrisol (Merck) and diluted, if necessary, to obtain solutions of lower concentration. Ultrapure filtered water (Milli-Q plus 185 water purification system) was employed in all experiments.

Voltammetric measurements by differential pulse polarography (DPP) and linear sweep voltammetry (LSV) were performed using a Metrohm-757 VA Computrace (Metrohm, Switzerland) attached to a personal computer with Computrace 9.0 data acquisition software also from Metrohm. Working electrodes were a multimode electrode Metrohm that can work either as a static mercury drop electrode (SMDE) or a hanging mercury drop electrode (HMDE) with a drop area of $0.9 \mathrm{~mm}^{2}$ and screen-printed carbon 
electrodes (SPCE) of $4 \mathrm{~mm}$ of diameter provided by DropSens (Spain). Double distilled $\mathrm{Hg}$ was used for SMDE or HMDE. Screen printed electrodes were connected to Metrohm-757 VA Computrace by means of a flexible cable (DropSens). Reference and auxiliary electrodes were, $\mathrm{Ag} / \mathrm{AgCl}, \mathrm{KCl}\left(3 \mathrm{~mol} \mathrm{~L}^{-1}\right)$, and glassy carbon respectively, all of them from Metrohm.

The instrumental parameters for polarographic experiments were a drop time of $1 \mathrm{~s}$, scan rate of $0.005 \mathrm{~V} \mathrm{~s}^{-1}$, pulse time of $0.05 \mathrm{~s}$, pulse amplitude of $0.05 \mathrm{~V}$, initial potential of $0.2 \mathrm{~V}$ and final potential of $-1.2 \mathrm{~V}$. The experimental parameters for LSV measurements were scan rate of $0.05 \mathrm{~V} \mathrm{~s}^{-1}$, initial potential of $0.2 \mathrm{~V}$ and final potential of -1.2 V. $\mathrm{pH}$ measurements were performed with a Crison $20 \mathrm{pH}$-meter. All the experiments were carried out at $20^{\circ} \mathrm{C}$ and purified nitrogen (Linde 50) was used for solution deaeration.

\subsection{Procedures}

Measurements were performed by DPP and LSV in a $0.02 \mathrm{~mol} \mathrm{~L}^{-1}$ TRIS buffer solution of $\mathrm{pH} 7.5$ also containing $0.05 \mathrm{~mol} \mathrm{~L}^{-1}$ of $\mathrm{KNO}_{3}$. In titrations of Cys with $\mathrm{Cd}(\mathrm{II}), 20 \mathrm{~mL}$ of $1.5 \times 10^{-5} \mathrm{~mol} \mathrm{~L}{ }^{-1}$ Cys solution in TRIS buffer were placed into the cell and purged with nitrogen during $20 \mathrm{~min}$. After that, aliquots of a $1 \times 10^{-3} \mathrm{~mol} \mathrm{~L}^{-1} \mathrm{Cd}(\mathrm{II})$ solution were added and DPP or LSV curves were recorded. Between additions, solutions were purged and mechanically stirred for $1 \mathrm{~min}$. Titrations were also performed in the opposite way, $20 \mathrm{~mL}$ of $1.5 \times 10^{-5} \mathrm{~mol} \mathrm{~L}^{-1} \mathrm{Cd}(\mathrm{II})$ solution were placed into the cell and, i.e., after deareation and measurement, aliquots of $1 \times 10^{-3} \mathrm{~mol} \mathrm{~L}^{-1}$ Cys solution were added, and the corresponding DPP or LSV curves were recorded. Data were corrected for dilution effects. 


\subsection{Data simulation for a 1:1 metal complex system}

A preliminary test of the proposed method has been carried out on simulated data. For this purpose, a data set was prepared with three overlapping signals, corresponding to the free ligand $\mathrm{L}$, the free metal ion $\mathrm{M}$ and a 1:1 ML complex with a stability constant of $\log K=6.0$. The total concentration of the metal was $1 \times 10^{-5} \mathrm{~mol} \mathrm{~L}^{-1}$ and the total ligand concentration ranged from 0 to $3 \times 10^{-5} \mathrm{~mol} \mathrm{~L}^{-1}$. A $5 \%$ of Gaussian noise was added to the simulated data. Table 1 summarises the values of the four EMG parameters used for each peak. In the case of 'b', their values were progressively changing for two of the peaks along the whole dataset to simulate the typical potential shift of many voltammetric signals.

\section{Results and discussion}

\subsection{Analysis of simulated data}

Fig. 3 illustrates the analysis of the data simulated as described in section 3.3 by using both EMG and GPA methods. In both cases, three components have been used to describe the system, corresponding to the metal ion, the metal complex and the free ligand (Fig. 3a). The application of EMG(-) algorithm was adjusted by least-squares to all the components producing an excellent data reproduction (Fig. 3b) with a very low lack of fit (lof) $1.1 \%$, whereas the adjustment of GPA method to the same simulated data yields a worse data reproduction (data not shown) with a higher lof of 9.9\%. In order to validate the applicability of EMG to this type of data, the theoretical and the adjusted concentration profiles from simulated data were compared. As it is shown in Fig. 3c, there is a good agreement between both theoretical and adjusted concentration profiles provided by EMG method. Moreover, as Table 1 shows, EMG fitting yields adjusted parameters very close to these used in the simulation. In contrast, the 
concentration profiles fitted by GPA method show a similar trend than theoretical ones but, as it can be expected, GPA is not able to adjust correctly the area of the strongly asymmetric voltammetric data (Fig. 3d). Therefore, at the view of the results, it can be concluded that EMG method is able to successfully adjust non-linear voltammetric data including strongly asymmetric and overlapping signals. Thus, the next step would be the application of the developed EMG method to an experimental system such as Cd(II)-Cys.

\subsection{Cd(II)-Cys system studied by DPP at SMDE}

Fig. 4a and $\mathrm{b}$ (black lines) show the current data matrix obtained in the DPP titration of Cd(II)-ion with cysteine (Cys) solution at pH 7.5 in $0.02 \mathrm{~mol} \mathrm{~L}^{-1}$ TRIS. As Cys is being added, the signal of free $\mathrm{Cd}(\mathrm{II})$ ion (component 2) starts to decrease and a new signal develops, which can be attributed to a Cd(II)-Cys complex (component 1) of 1:2 stoichiometry [15]. Finally, at large enough concentrations of added Cys, the peak related with the anodic signal of free Cys starts to increase (component 3). A visual inspection of the experimental data matrix evidences an important movement of signal 1 which also shows a strong asymmetry with a pronounced left queue. As for signal 3, it is slightly asymmetric and still has a slight shift, whereas signal 2 remains practically at the same potential and is quite symmetric. These facts, which can be observed in detail in inset of Fig. 4a for some selected voltammograms, are consistent with the electrochemical reversibility of $\mathrm{Cd}(\mathrm{II})$ reduction, in contrast with the irreversible character of both the reduction of the inert $\mathrm{Cd}(\mathrm{II})$-Cys complex and the anodic oxidation of the free Cys. These behaviours suggest a decrease in the bilinearity of the data. Thus, the application of MCR-ALS is not appropriate since a certain rotational ambiguity in the attribution of the pure spectrum to each component is observed and more 
components are required to explain this lack of linearity [5-8, 16-17]. At the view of both non-bilinearity of the data and the strong asymmetry we applied the developed PSF-EMG method to this Cd(II)-Cys system and compared the obtained results with those computed by the simplest PSF strategy (GPA), based on the fitting of two gaussian equations to each voltammetric peak $[5,7]$.

As it can be seen in Fig. 4a (red lines) the use of this EMG method produces an excellent data reproduction with the three previously described components, i.e., $\mathrm{Cd}(\mathrm{II})$ complex, free Cd(II) ion and free Cys. In this case, EMG (+, left queue) algorithm was adjusted by least-squares to component 2 whereas EMG (-, right queue) was perfectly fitted to components 1 and 3 . In contrast, the reproduced data provided by the application of GPA method to the experimental data shows a lack of fit especially visible in components 1 and 3 (Fig. 4b, red lines). Then, the strong asymmetry observed in these signals hinders the adjustment of the two gaussian functions. Thus, it is clear that the EMG method produces a very good lof as compared to GPA which cannot completely adjust these non-gaussian signals (Table 2). It is interesting to compare the EMG results with these obtained through the ALPA algorithm [8]. The data reproduction is also very good (not shown) and the lack of fit is slightly higher, as Table 2 shows.

When EMG method is applied to current matrix obtained from direct titration the concentration profiles (Fig. 4c) show that free Cd(II) signal (component 2) decreases with cysteine additions until reaching Cys:Cd(II) ratio of 2 , when it stabilizes indicating the formation of $\mathrm{ML}_{2}$ complex. With the first cysteine additions the component 1 related to $\mathrm{Cd}(\mathrm{II})$-complex signal increases until the ratio value of 2 and then remains constant. Regarding the free cysteine (component 3), it increases from ratio 2 confirming the formation of the $\mathrm{ML}_{2}$ complex. The concentration profiles obtained by 
ALPA (not shown) are practically the same. These results are in good agreement with previous ones published in the literature [15]. When GPA method is applied to the same data matrix the obtained concentration profiles (Fig. 4d) show similar results regarding the behaviour of the free $\mathrm{Cd}(\mathrm{II})$ and free Cys signals. However, component 1 related with the $\mathrm{Cd}(\mathrm{II})$-complex signal appears and increases from the first addition but this signal reaches a maximum at ratio $1 \mathrm{Cys}$ :Cd erroneously indicating the formation of only the ML complex. This misleading concentration profile is due to the adjustment of the gaussian equations to strongly asymmetric peaks which provides a lack of the fitting in the vicinity of the maximum of the signal. This drawback of the GPA application to this kind of signal can be also seen in the obtained lof after the least squares iterative process.

Taking into consideration the abovementioned problems related to the asymmetry of the voltammetric signals, DPP titration of Cys with $\mathrm{Cd}(\mathrm{II})$ solution at the same mentioned conditions were also considered in order to corroborate the robustness of EMG method. The obtained experimental matrix of the reverse titration is shown in Fig. 5a, which illustrates three different signals. Initially, only the free Cys signal (component 3) is present. However, when $\mathrm{Cd}(\mathrm{II})$ is added, two signals (components 2 and 1) appear which are related to the $\mathrm{Cd}(\mathrm{II})$-complex formed in solution and the free $\mathrm{Cd}(\mathrm{II})$, respectively. As it can be seen the reverse titration provides more intricate voltammetric data than those obtained in the direct titration. Component 1 presents not only a potential shift and a pronounced asymmetry but also is strongly overlapped with component 2. Despite of the lack of linearity, the asymmetry of the peaks and the overlapping observed, the application of EMG (+, left queue) algorithm to component 2 and EMG (-, right queue) to components 1 and 3 provides the concentration profiles with a very low lack of fit (5.8\%). ALPA can be also applied with similar results and 
with a slightly higher lof (6.8\%). In contrast, the application of GPA method to the same voltammetric data resulted in a too large lof $(19.3 \%)$.

Taking advantage of the peak-shaped adjustment, the EMG program yields a concentration profiles matrix from the fitted peak areas (Fig. 5b). As it can be seen, the concentration of free Cys (component 3) decreases until its disappearance at ratio 0.5 indicating the formation of the $\mathrm{ML}_{2}$ complex. With the first $\mathrm{Cd}(\mathrm{II})$ additions component 1 corresponding to the Cd-complex signal increases until the ratio 0.5 and then it stabilizes. The formation of $\mathrm{ML}_{2}$ complex is also confirmed by the appearance of the free Cd(II) signal (component 2) at the same ratio. The application of GPA method to these experimental data produces similar concentration profiles (Fig. 5c) but also certain ambiguities related with the emergence of free $\mathrm{Cd}(\mathrm{II})$ signal and the stabilization of $\mathrm{Cd}(\mathrm{II})$-complex signal which take place a ratio higher than 0.5 can be observed. This anomalous behaviour could be attributed to the strong asymmetry combined with the overlapping of the signals.

The successful application of EMG method to reverse titration of the system Cd:Cys corroborates the goodness of this method for the adjustment of non-gaussian peak-shape in non-linear and overlapped voltammetric data reaching the expected complexation model.

\subsection{Cd(II)-Cys system studied by LSV at SPCE}

The high toxicity of the soluble salts of mercury as well as the emergence of the screenprinting technology as a valuable alternative to mercury electrodes for the electrochemical and chemometric study of metal complexation by thiol-rich peptides [18] lead us to use for the first time a screen-printed carbon electrode (SPCE) instead of SMDE for the study of Cd(II):Cys system. 
The titration of Cys with Cd(II) solution (reverse titration) was carried out by LSV in SPCE at $50 \mathrm{mV} \mathrm{s}^{-1}$. In contrast with mercury electrodes, carbon electrodes do not produce anodic signals for free Cys in the potential region studied (unlike mercury, carbon is not easily oxidised by thiols to form highly stable sulphides). As a consequence, the voltammetric data obtained were relatively simple, with only two signals (Fig. 6a, black lines), one related with the Cd-complex at $c a .-0.7 \mathrm{~V}$ and other signal at $c a$. $-1.1 \mathrm{~V}$ corresponding to the free $\mathrm{Cd}(\mathrm{II})$ which, although it is expected to be peak-shaped, is strongly overlapping with the exponential growth of the baseline caused by the reduction of the solvent. For this reason it is interesting to evaluate the capability of the EMG method to analyse these data, since neither GPA nor ALPA methods are able to adjust the exponential-like signal strongly overlapping with the background current [19]. It is true that MCR-ALS could explain the evolution of the baseline by taking it as a component, but the large potential shifts of the signals would produce a considerable error.

As it can be seen in Fig. 6a (red lines) the use of EMG method produces a good data reproduction with a very low lof of $3.2 \%$. In this adjustment, EMG (-, right queue) algorithm was fitted by least-squares to component 2 whereas EMG (+, left queue) was perfectly adjusted to the intricate combination of a small peak overlapped to a fast growing baseline (components 1). Indeed, EMG fitting seems to consider this last signal as the queue of a very high peak. In contrast, the reproduced data provided by GPA method in Fig. 6b (red lines) show a good adjustment of the $\mathrm{Cd}(\mathrm{II})$-complex peak which shifts and changes the width, but as mentioned above it is not able to fit the strongly overlapping peak (Inset Fig. 6b) producing a lack of fit of $10.8 \%$.

When EMG method is applied to the experimental matrix, the resulting concentration profiles (Fig. 6c) show that from the first Cd(II) addition the component 2 related to 
Cd(II)-complex signal increases until the ratio value of 0.5 and then remains constant, indicating the formation of the $\mathrm{ML}_{2}$ complex. Also from this ratio, the free $\mathrm{Cd}(\mathrm{II})$ signal (component 1), appears and increases confirming the formation of the $\mathrm{ML}_{2}$ complex. However, when GPA method is applied to the data matrix from Fig. 6b (black lines), the concentration profiles presented in Fig. 6d are obtained with a higher lof $(10.8 \%)$. As it is shown in concentration profiles, the signal of $\mathrm{Cd}(\mathrm{II})$-complex (component 2) has a similar behaviour than that obtained in previous applications of the EMG method, increasing from the first addition until reaching the $0.5 \mathrm{Cd}(\mathrm{II})$ :Cys ratio. Nevertheless, an incoherent profile is obtained for the free $\mathrm{Cd}(\mathrm{II})$ signal. Hence, it can be concluded that SPCE provides similar results as those provided by mercury electrodes in the application of the EMG method to the complexation study of $\mathrm{Cd}(\mathrm{II})$ :Cys system.

\section{Conclusions}

The exponentially modified gaussian (EMG) method appears to be a promising strategy for the multivariate curve resolution of non-linear voltammetric data with highly asymmetric and strongly overlapped signals. For this purpose two algorithms EMG(-) and $\mathrm{EMG}(+)$ are able to adjust by least-squares the queue at the right or left side of the maximum of the signals, respectively.

From the comparison of EMG and GPA fittings in the considered systems, it can be concluded that concentration profiles yielded by both methods present a similar trend, but EMG produces a more satisfactory data reproduction than that obtained by GPA, since EMG fitting is more accurate in the peak region closer to maximum.

As compared to ALPA, the previous PSF approach for highly asymmetric signals based on the asymmetric logistic function, the fitting ability of the EMG method is slightly better, with lower values for the lack of fit. By other hand, EMG can deal with 
exponential baselines and produces a more robust fitting than ALPA, where iterations frequently fail unless a very accurate initial estimation set is provided. Moreover, EMG directly fits the peak area as one of the adjustable parameters, which can be useful in the study of electrochemical non-reversible systems. Thus, EMG constitutes a significant improvement as compared to ALPA and, especially, as compared to GPA.

Finally, for the first time a non-toxic SPCE was used instead of mercury electrode for the study of $\mathrm{Cd}(\mathrm{II})$ :Cys system. Although this electrode provides more intricate signals, the application of EMG method to this voltammetric data allows, unlike previous GPA and ALPA approaches, the successful resolution of this system with a low lof. The concentration profiles obtained permit the establishment of a complexation model which is in agreement with those obtained by mercury electrode and published in the literature. This encourages the study of heavy metal ion complexation with alternative electrodes, such as SPCE.

\section{Acknowledgments}

This work is supported by the Ministry of Science and Innovation of Spain (Project CTQ2012-32863) and the Generalitat of Catalonia (Project 2014SGR269). 


\section{References}

[1] A.J. Bard, L.R. Faulkner, Electrochemical Methods: Fundamentals and Applications, 2nd ed., Wiley, New York, 2001.

[2] E. Richards, C. Bessant, S. Saini, Multivariate data analysis in electroanalytical chemistry, Electroanalysis, 14 (2002) 1533-1542.

[3] M. Esteban, C. Ariño, J.M. Díaz-Cruz, Chemometrics in electroanalytical chemistry, Crit. Rev. Anal. Chem. 36 (2006) 295-313.

[4] X. Cetó, F. Céspedes, M. del Valle, Comparison of methods for the processing of voltammetric electronic tongues data, Microchim. Acta 180 (2013) 319-330.

[5] S. Cavanillas, C. Ariño, J.M. Díaz-Cruz, M. Esteban, Parametric signal fitting by gaussian peak adjustment: a new multivariate curve resolution method for nonbilinear voltammetric measurements, Anal. Chim. Acta 689 (2011) 198-205.

[6] J.M. Díaz-Cruz, A. Alberich, C. Ariño, M. Esteban, Three-dimensional voltammetry assisted by parametric signal fitting: a new perspective for the electrochemical evaluation of metal binding in the presence of electrodic adsorption, Anal. Chim. Acta 777 (2013) 17-24.

[7] S. Cavanillas, N. Serrano, J.M. Díaz-Cruz, C. Ariño, M. Esteban, Parametric signal fitting by gaussian peak adjustment: Implementation of 2D transversal constraints and its application for the determination of $\mathrm{pKa}$ and complexation constants by differential pulse voltammetry, Analyst 138 (2013) 2171-2180.

[8] M. Kooshki, J.M. Díaz-Cruz, H. Abdollahi, C. Ariño, M. Esteban, Asymmetric logistic peak as a suitable function for the resolution of highly asymmetric voltammograms in non-bilinear systems, Analyst 136 (2011) 4696-4703. 
[9] J.P. Foley, J.G. Dorsey, A Review of the exponentially modified Gaussian (EMG) function: Evaluation and subsequent calculation of universal data, J. Chromatogr. Sci. 22 (1984) 40-46.

[10] M.S. Jeansonne, J.P. Foley, Review of the exponentially modified Gaussian (EMG) function Since 1983, J. Chromatogr. Sci. 29 (1991) 258-266.

[11] K. Lan, J.W. Jorgenson, A hybrid of exponential and Gaussian functions as a simple model of asymmetric chromatographic peaks, J. Chromatogr. A 915 (2001) 1-13.

[12] Y. Kalambet, Y. Kozmin, K. Mikhailova, I. Nagaev, P. Tikhonov, Reconstruction of chromatographic peaks using the exponentially modified Gaussian function, J. Chemometrics 25 (2011) 352-356.

[13] Matlab, version R2008b, MathWorks, Inc., Natick, MA, USA, 2008.

[14] J.M. Díaz-Cruz, J. Agulló, M.S. Díaz-Cruz, C. Ariño, M. Esteban, R. Tauler, Implementation of a chemical equilibrium constraint in the multivariate curve resolution of voltammograms from systems with successive metal complexes, Analyst 126 (2001) 371-377.

[15] R. Gusmão, C. Ariño, J.M. Díaz-Cruz, M. Esteban, Competitive binding of cadmium by plant thiols: an electrochemical study assisted by multivariate curve resolution, Anal. Bioanal. Chem. 394 (2009) 1137-1145.

[16] A. Alberich, J.M. Diaz-Cruz, C. Ariño, M. Esteban, Potential shift correction in multivariate curve resolution of voltammetric data. General formulation and application to some experimental systems, Analyst 133 (2008) 112-125.

[17] A. Alberich, J.M. Diaz-Cruz, C. Ariño, M. Esteban, Combined use of the potential shift correction and the simultaneous treatment of spectroscopic and 
electrochemical data by multivariate curve resolution: Analysis of a $\mathrm{Pb}$ (II)phytochelatin system, Analyst 133 (2008) 470-477.

[18] V. Sosa, N. Serrano, C. Ariño, J.M. Díaz-Cruz, M. Esteban, Can bismuth film screen printed carbon electrodes be used to study complexation? Talanta 107 (2013) 356-360.

[19] S. Cavanillas, E. Chekmeneva, C Ariño, J.M. Diaz-Cruz, M. Esteban, Electroanalytical survey of the As(III) complexation by the poisoning remediators 2,3-dimercaptopropane-1-sulfonate (DMPS) and meso- 2,3-dimercaptosuccinic acid (DMSA), Anal. Chim. Acta 746 (2012) 47-52. 


\section{Figure Captions}

Figure 1. Comparison of simulated voltammograms using GPA (1), EMG(-) (2) and $\operatorname{EMG}(+)(3)$. The parameters used in the simulation were $a=0.9, b=0.6, c=500, d=150$ for GPA and $a=0.1, b=0.6, c=0.02, d=0.07$ for both EMG functions.

Figure 2. Flowchart of the proposed PSF-EMG method.

Figure 3. Simulation of voltammetric data for a system including a free metal ion (2), a free ligand (1) and a 1:1 complex (3) by using the parameters shown in Table 1. Comparison between simulated voltammograms (a) and signals adjusted by means of the proposed EMG method (b), and between simulated and adjusted concentration profiles by EMG method (c) and GPA method (d). Simulated profiles are shown in solid lines and adjusted values are denoted by points.

Figure 4. Comparison between the experimental DPP voltammograms obtained in the titration of $1 \times 10^{-5} \mathrm{~mol} \mathrm{~L}^{-1} \mathrm{Cd}(\mathrm{II})$ solution with Cys using a SMDE at $\mathrm{pH} 7.5$ and ionic strength $0.05 \mathrm{~mol} \mathrm{~L}^{-1} \mathrm{KNO}_{3}$ (a and $\mathrm{b}$, black lines) and reproduced data yield by the proposed EMG method (a, red lines) and GPA method (b, red lines). Concentration profiles obtained by EMG method (c) and GPA method (d).

Components assignments: 1 - Cd(II)-complex; 2 - free Cd(II)-ion; 3 - free cysteine. 
Figure 5. Experimental DPP voltammograms obtained in the titration of $1 \times 10^{-5} \mathrm{~mol} \mathrm{~L}^{-}$ ${ }^{1}$ Cys solution with $\mathrm{Cd}(\mathrm{II})$ using a SMDE at $\mathrm{pH} 7.5$ and ionic strength $0.05 \mathrm{~mol} \mathrm{~L}^{-1}$ $\mathrm{KNO}_{3}$ (a). Concentration profiles obtained by EMG method (b) and GPA method (c). Components assignments: 1 - Cd(II)-complex; 2 - free Cd(II)-ion; 3 - free cysteine.

Figure 6. Comparison between the experimental LSV voltammograms obtained in the titration of $1 \times 10^{-5} \mathrm{~mol} \mathrm{~L}^{-1}$ Cys solution with Cd(II) using a SPCE at $\mathrm{pH} 7.5$ and ionic strength $0.05 \mathrm{~mol} \mathrm{~L}^{-1} \mathrm{KNO}_{3}$ (a and b, black lines) and reproduced data yield by the proposed EMG method (a, red lines) and GPA method (b, red lines). Concentration profiles obtained by EMG method (c) and GPA method (d).

Components assignments: 1 - free Cd(II)-ion; 2 - Cd(II)-complex. 
Table 1. Comparison of the parameters fitted by EMG method with the parameters of Eqn. (1) used for the simulation of the voltammetric signals of the free ligand (peak 1), the free metal (peak 2) and a 1:1 complex (peak 3) with a stability constant of $\log K=$ 6.0. The total concentration of metal was $1 \times 10^{-5}$ mol $\mathrm{L}^{-1}$ and the total ligand concentration ranged from 0 to $3 \times 10^{-5} \mathrm{~mol} \mathrm{~L}^{-1}$. Simulated voltammograms at every total ligand concentration were obtained as the sum of the peak of every species multiplied by its equilibrium concentration and divided by the total metal concentration. For comparison purposes, the values of the parameters adjusted in the fitting are also given.

\begin{tabular}{cccc}
\hline \hline Parameters simulation & Ligand (peak 1) & Metal (peak 2) & Complex (peak 3) \\
\hline $\mathrm{a}$ & 0.1 & 0.1 & 0.1 \\
$\mathrm{~b}$ & $0.230-0.330$ & $0.400-0.430$ & 0.500 \\
$\mathrm{c}$ & 0.02 & 0.03 & 0.03 \\
$\mathrm{~d}$ & 0.05 & 0.02 & 0.04 \\
Parameters fitted by EMG & & & \\
\hline $\mathrm{a}$ & Their values were progressively changing with L:M ratio, see in Fig. 3c. \\
$\mathrm{b}$ & $0.268-0.331$ & $0.402-0.437$ & $0.495-0.500$ \\
$\mathrm{c}$ & 0.02 & $0.03-0.04$ & 0.03 \\
$\mathrm{~d}$ & 0.05 & $0.01-0.03$ & 0.04 \\
\hline \hline
\end{tabular}


Table 2. Comparison of the values of lack of fit obtained by applying GPA, ALPA and EMG methods to the voltammetric data sets shown in Figures 4-6.

\begin{tabular}{lccc}
\hline \hline Titration & GPA & ALPA & EMG \\
\hline Cys to Cd(II) by DPP at Hg electrode & $19,1 \%$ & $5.5 \%$ & $5.2 \%$ \\
Cd(II) to Cys by DPP at Hg electrode & $19.3 \%$ & $6.8 \%$ & $5.8 \%$ \\
Cd(II) to Cys by LSV at SPC electrode & $10.8 \%$ & - & $3.2 \%$ \\
\hline \hline
\end{tabular}


Figure 1
Click here to download high resolution image

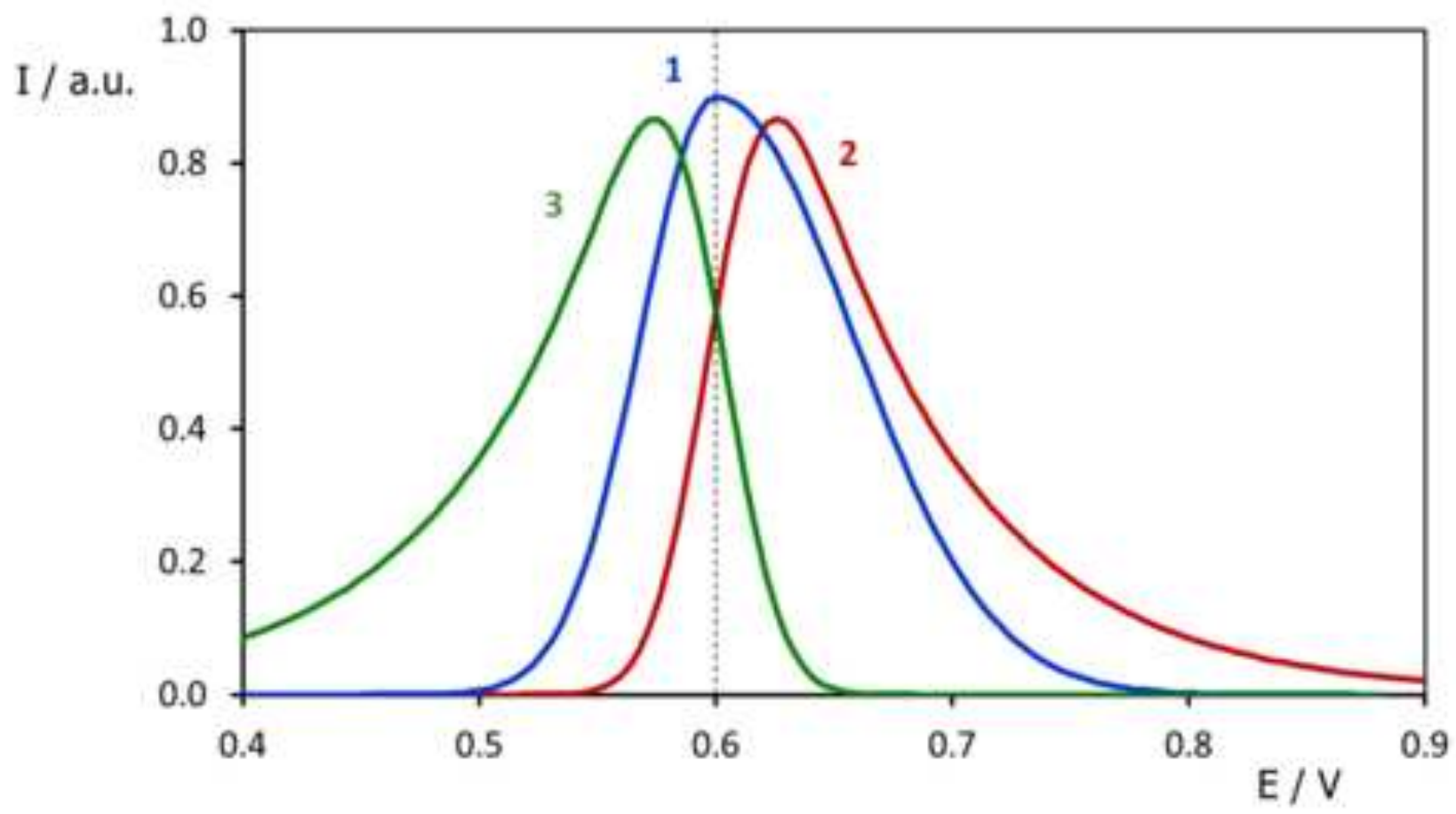

1: GPA function with $a=0.9, b=0.6, c=500, d=150$

2: EMG- function with $a=0.1, b=0.6, c=0.02, d=0.07$

3: EMG+ function with $a=0.1, b=0.6, c=0.02, d=0.07$ 

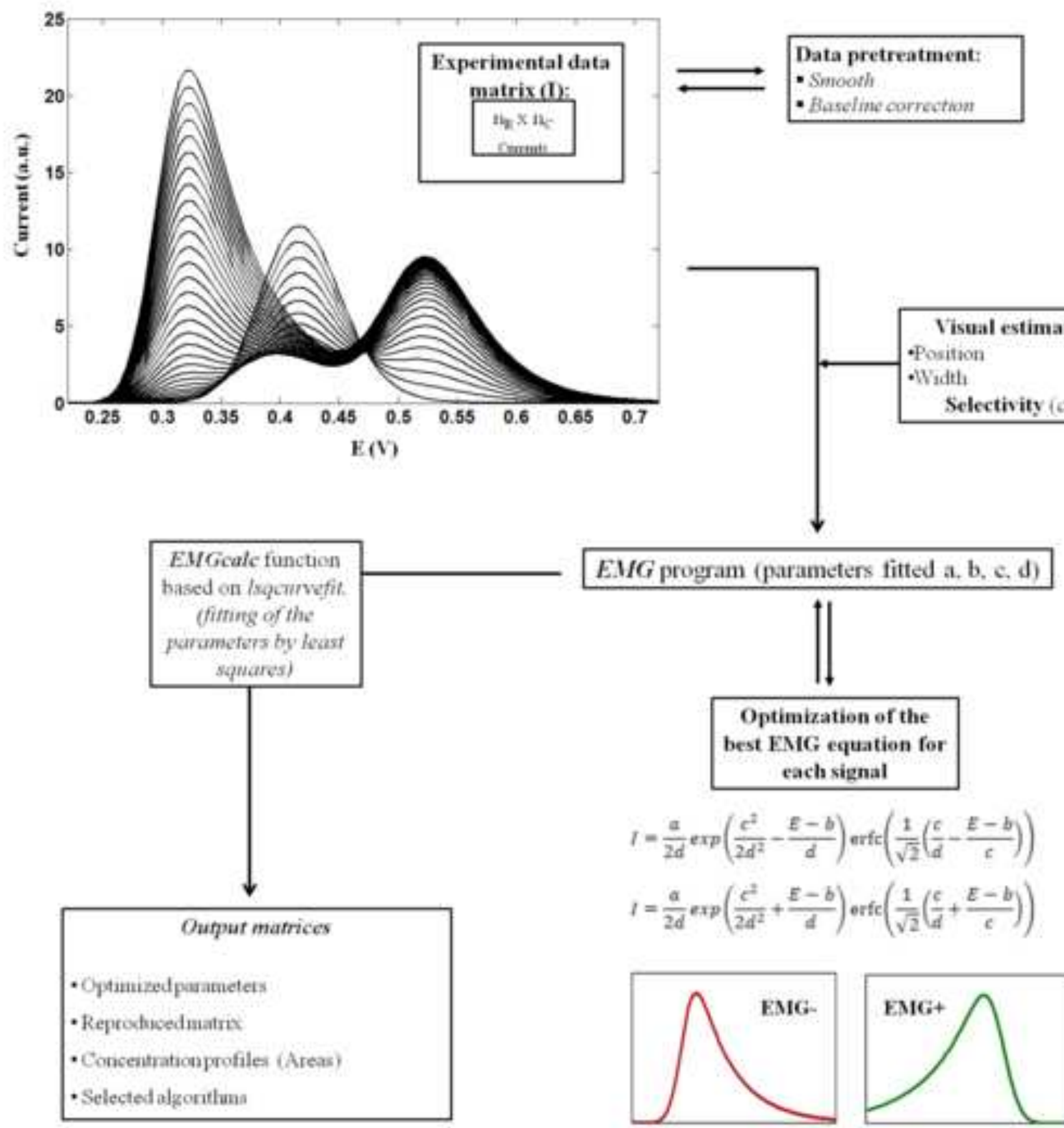

Vistual estimation: Position

- Wadth

Selectivity $(e s e h)$

\section{Figure 2
Click here to download high resolution image}

E(V)

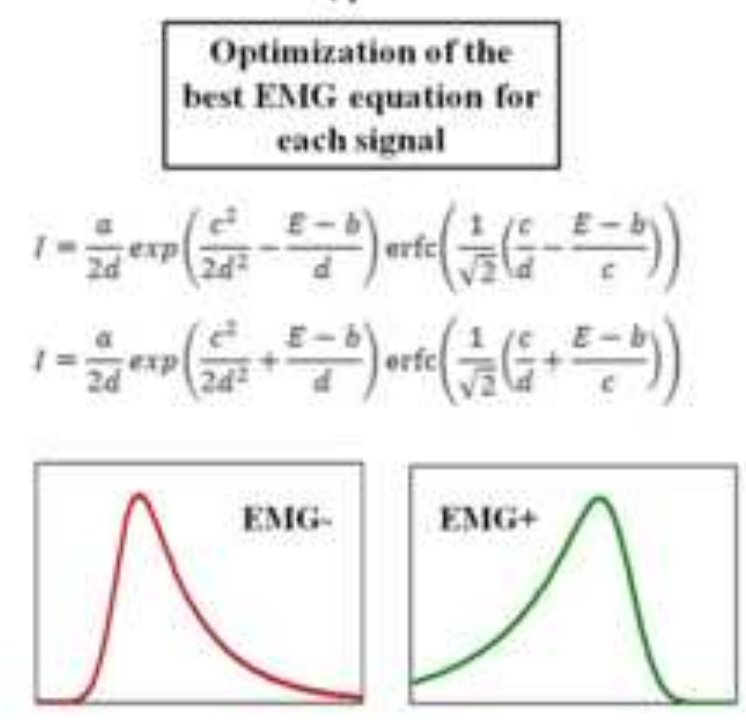

Optimization of the
best EMG equation for each signal

$$
\begin{aligned}
& t=\frac{a}{2 d} \exp \left(\frac{c^{2}}{2 d^{2}}-\frac{E-b}{d}\right) \operatorname{erfc}\left(\frac{1}{\sqrt{2}}\left(\frac{c}{d}-\frac{E-b}{c}\right)\right) \\
& I=\frac{a}{2 d} \exp \left(\frac{c^{2}}{2 d^{2}}+\frac{E-b}{d}\right) \operatorname{erfc}\left(\frac{1}{\sqrt{2}}\left(\frac{c}{d}+\frac{E-b}{c}\right)\right)
\end{aligned}
$$


Figure 3

Click here to download high resolution image

a)

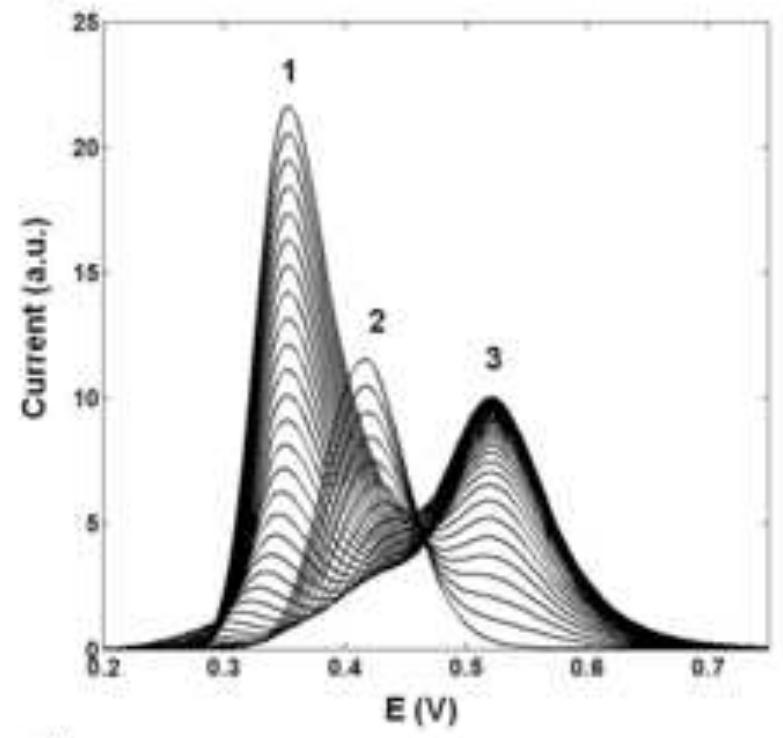

b)

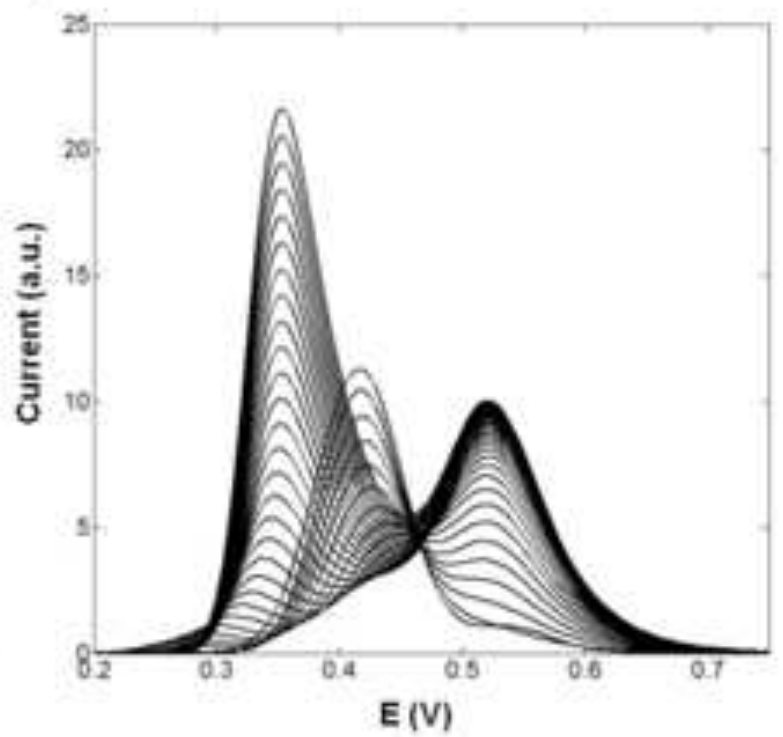

c)

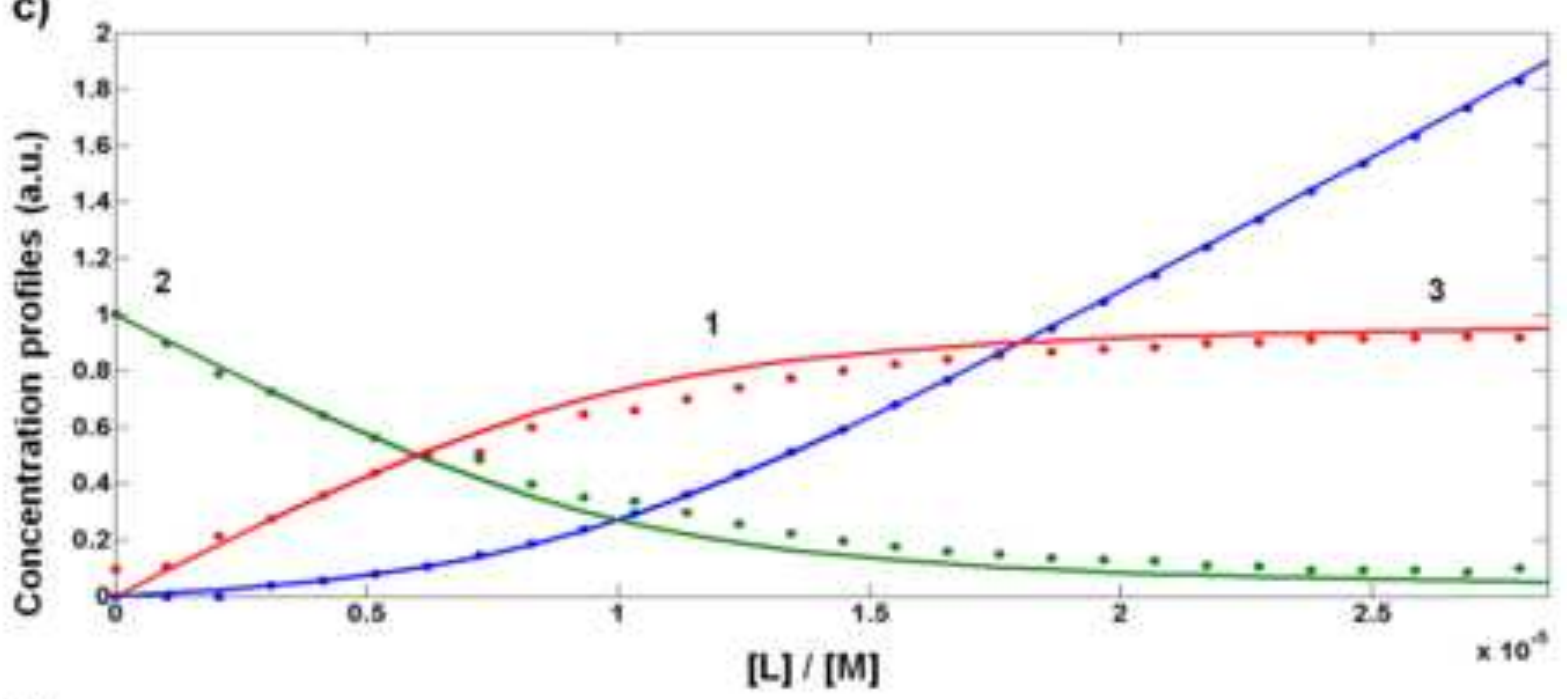

d)

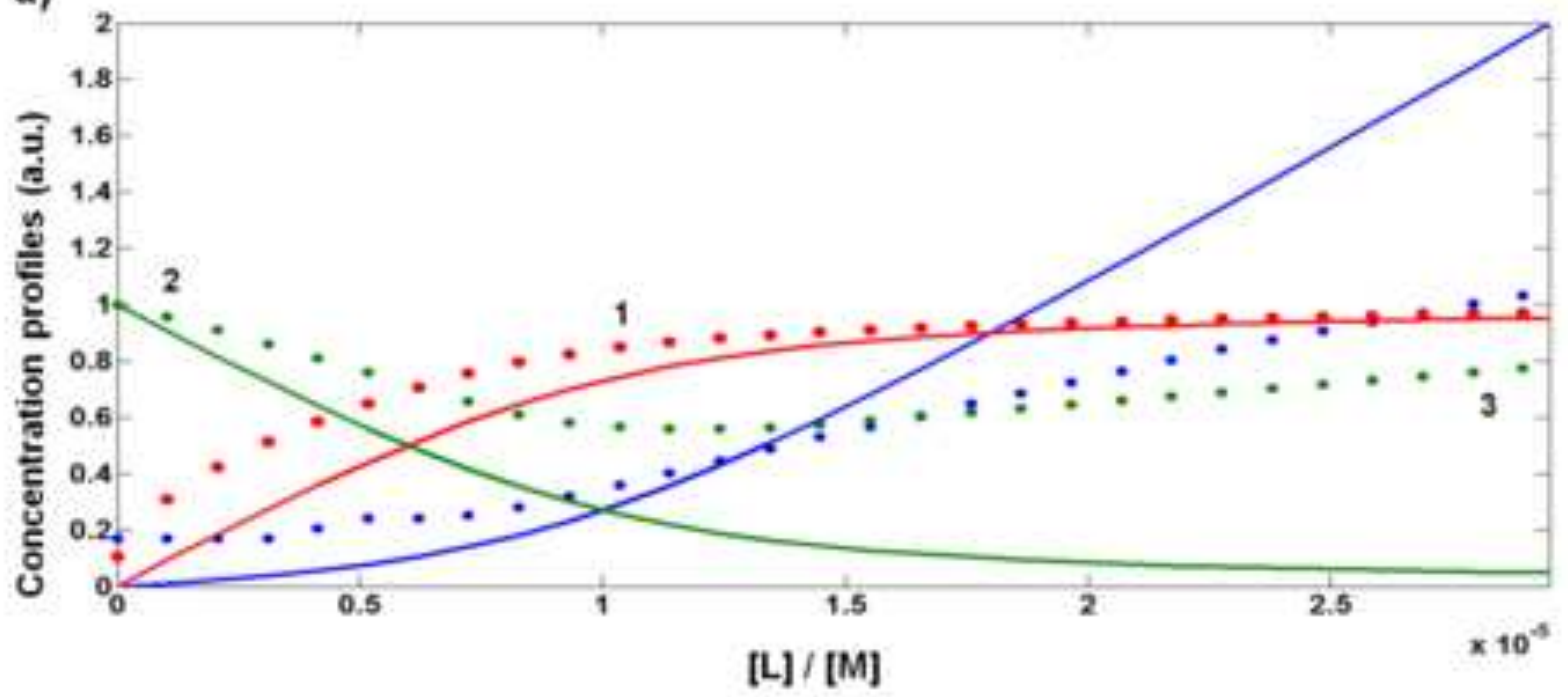

$[$ [L] $/[\mathrm{M}]$ 


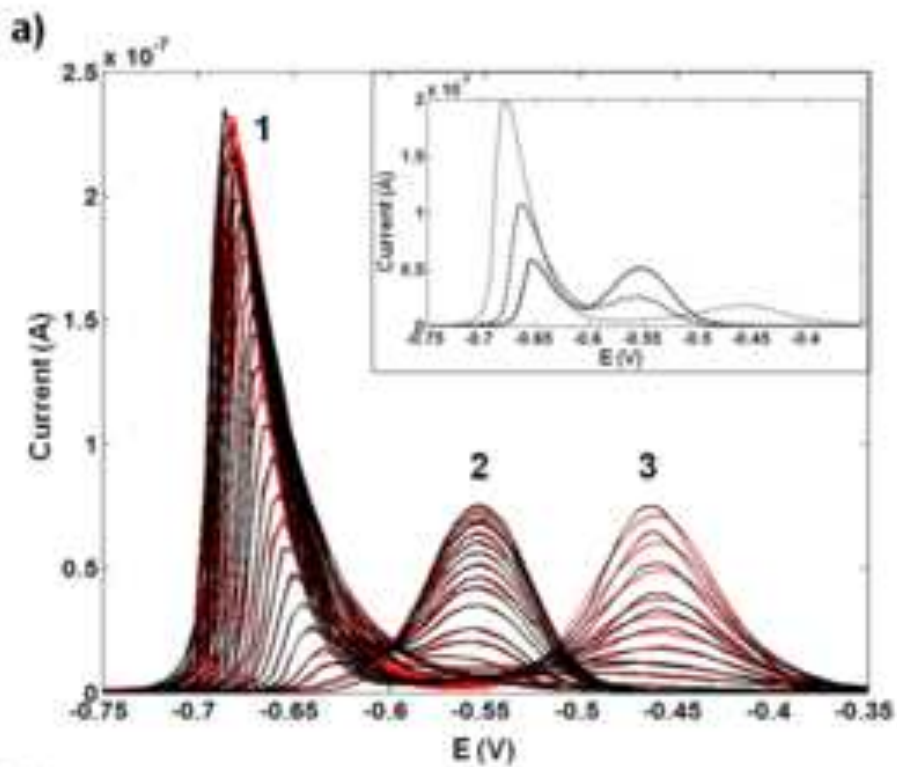

c)

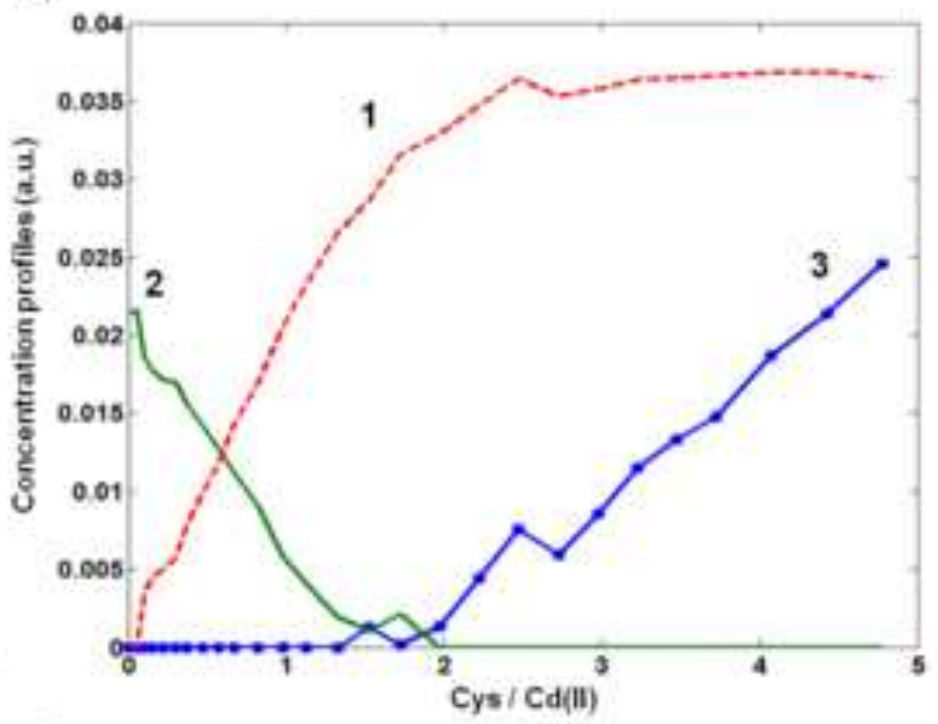

b)

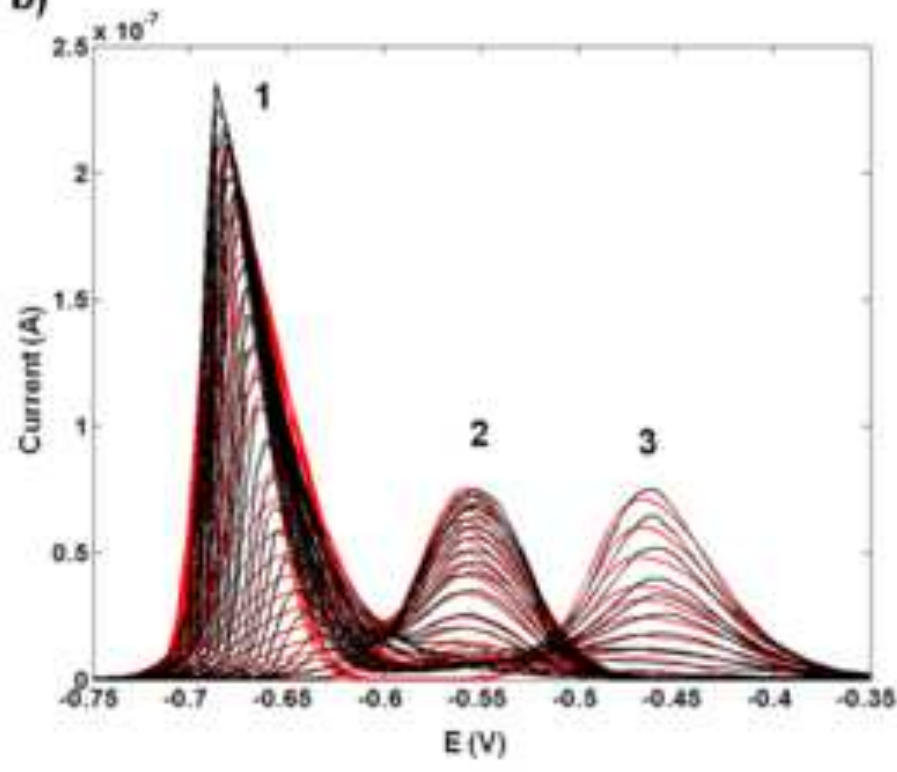

d)

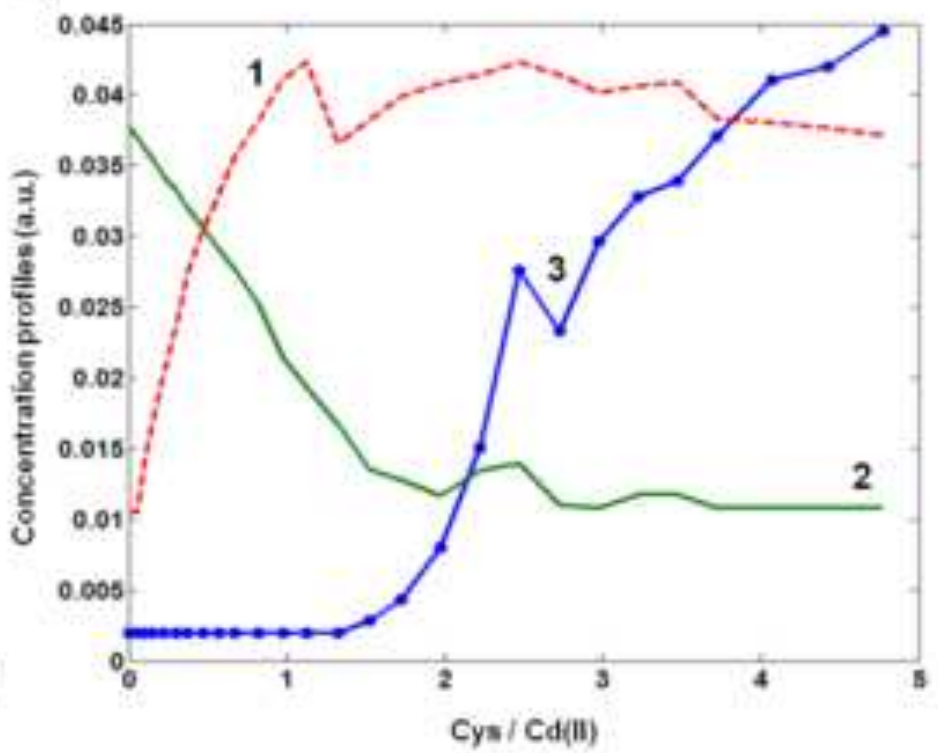




\section{Fure 5}

Click here to download high resolution image

a)

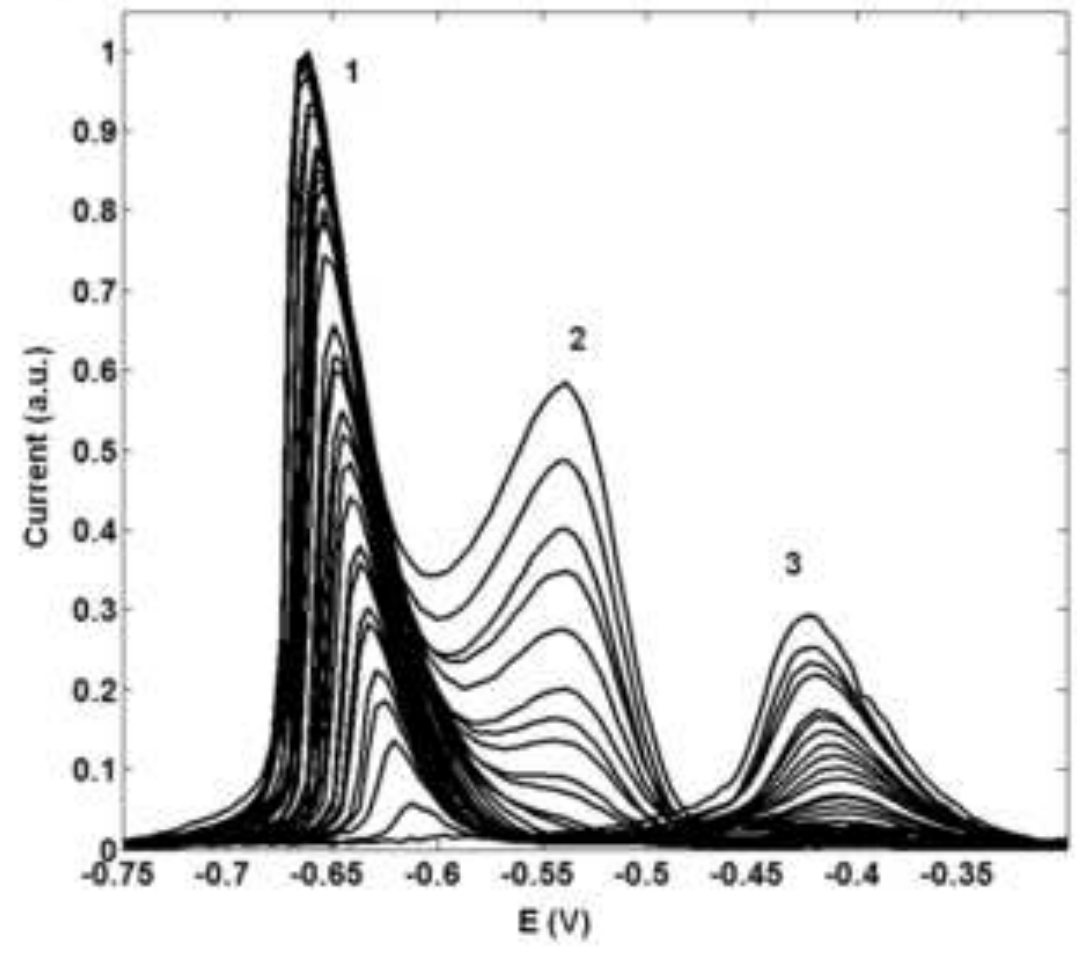

b)
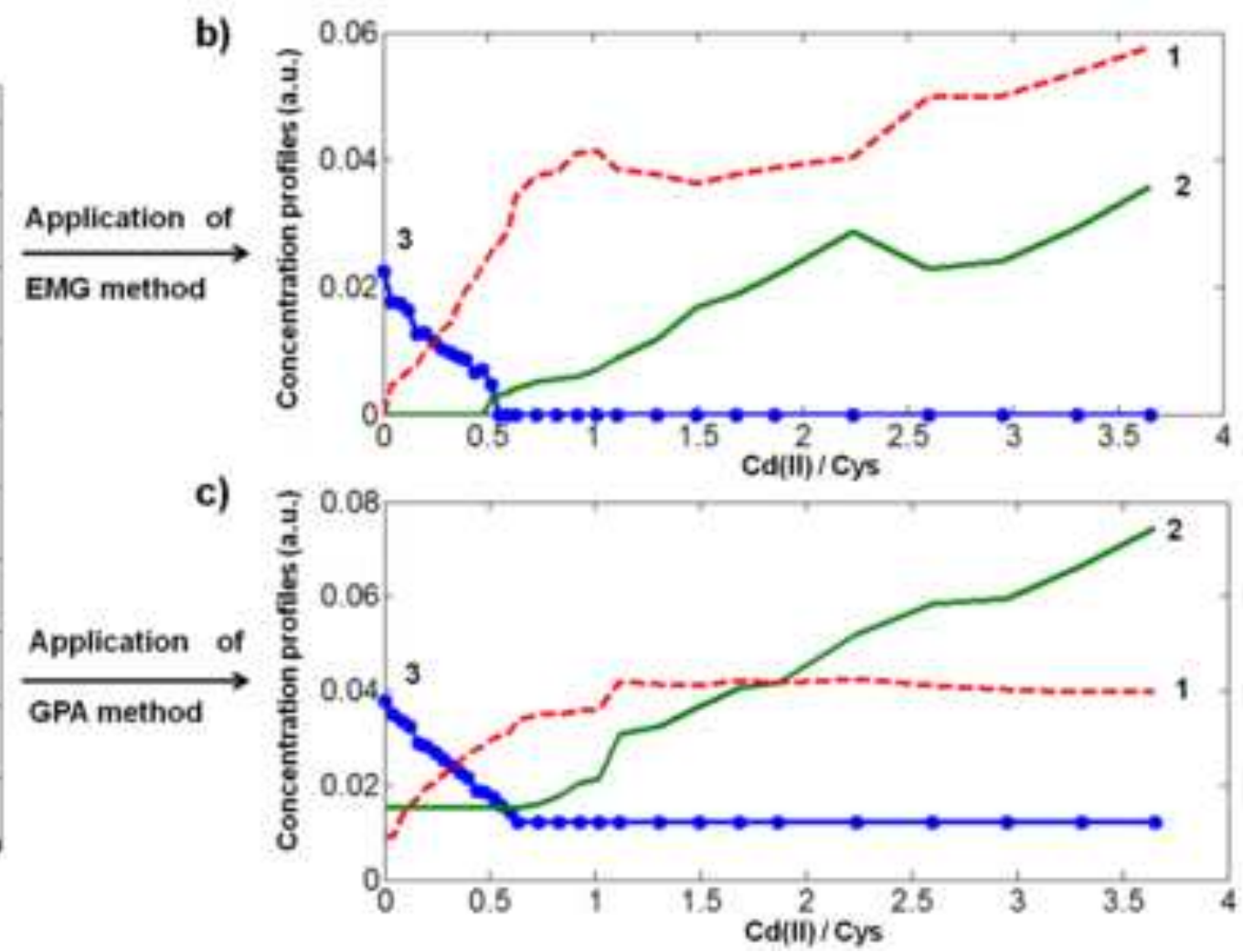
a)

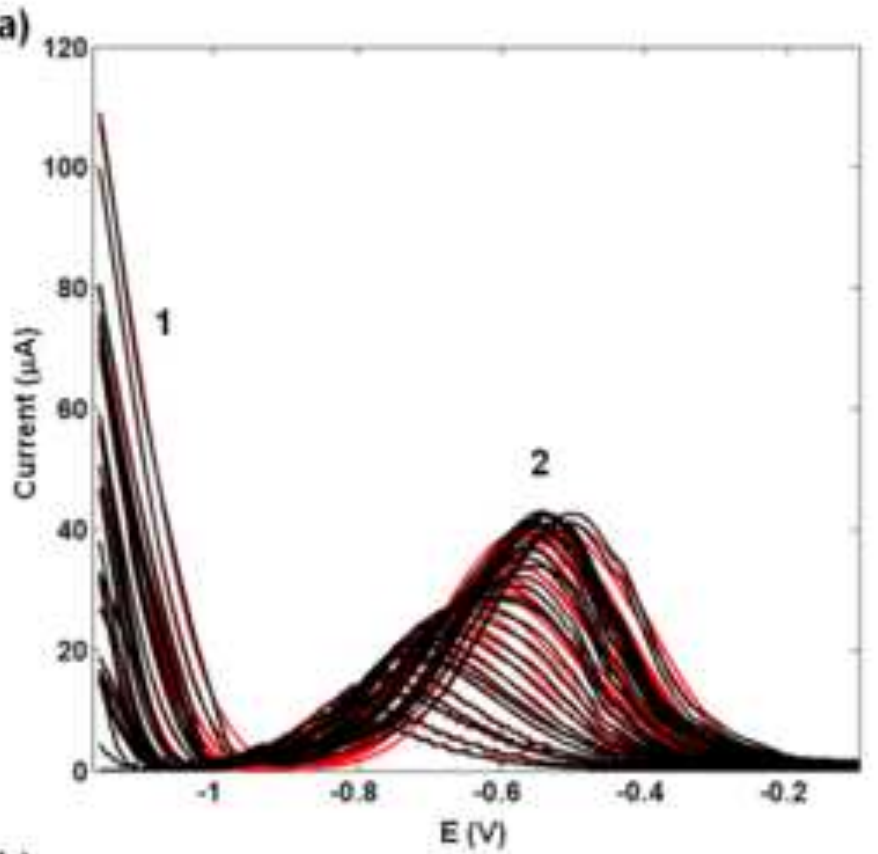

b)

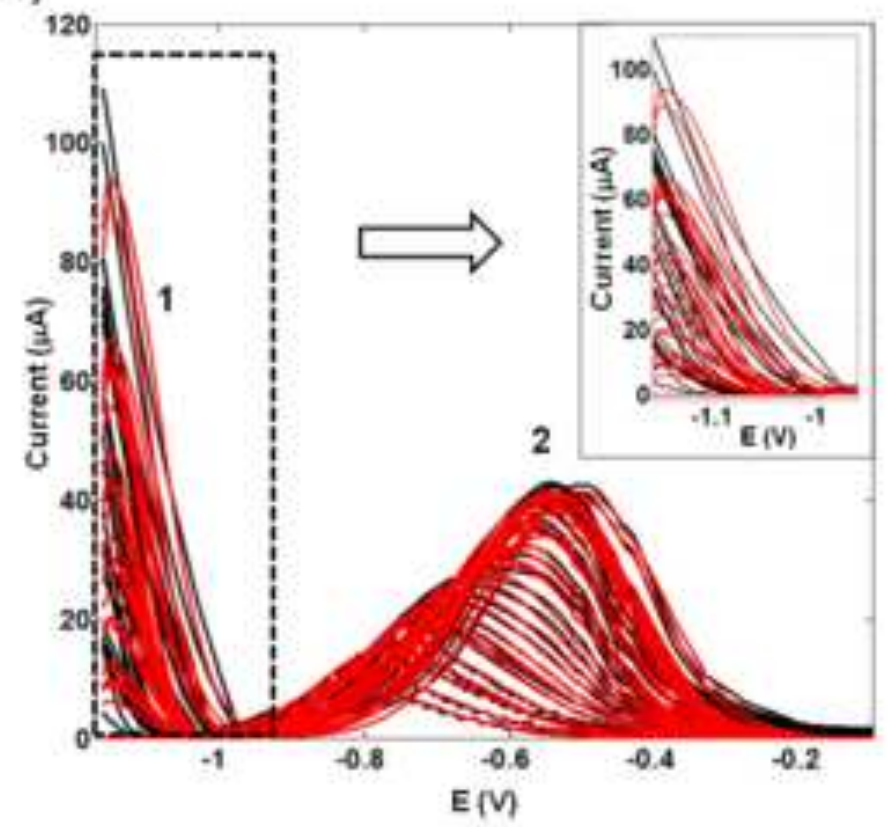

c)

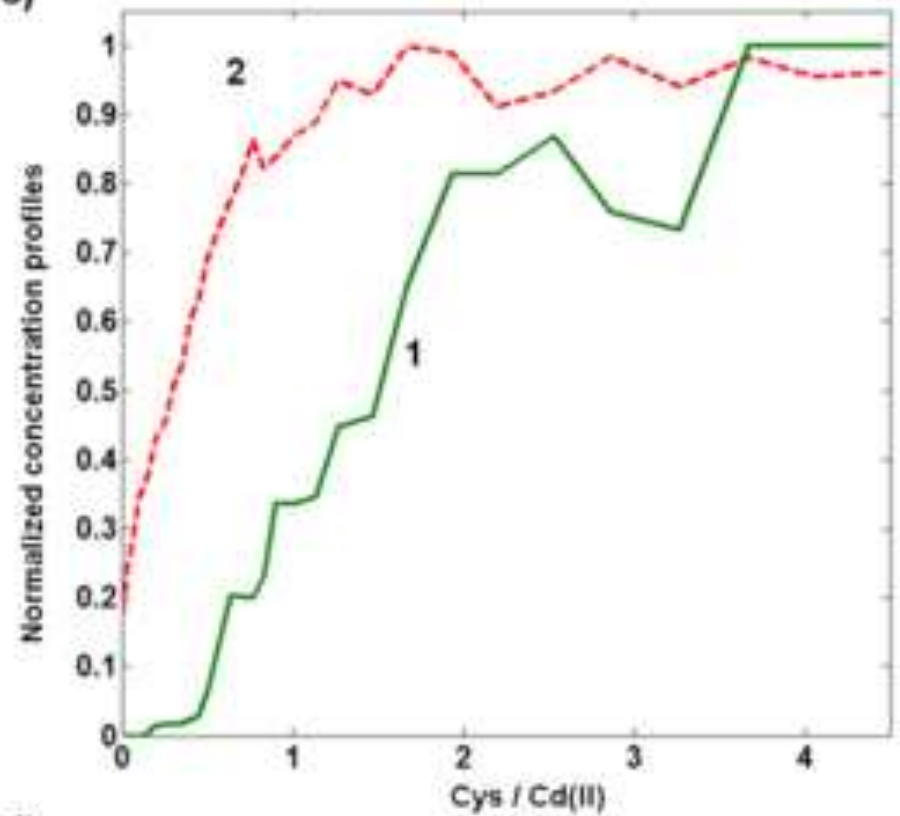

d)

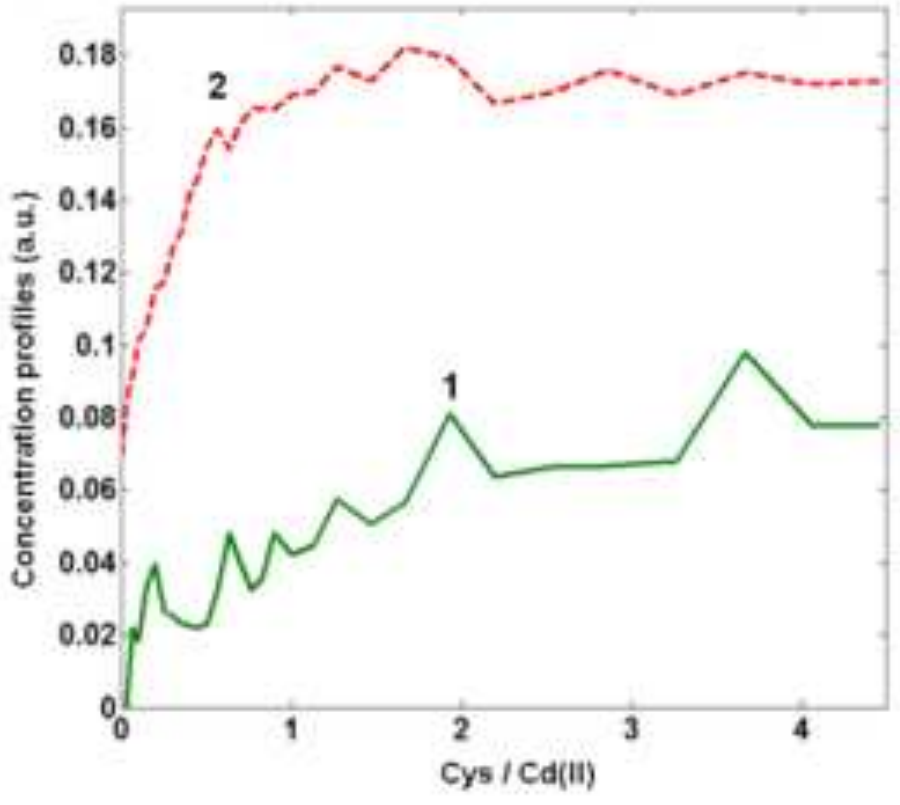

